\title{
Urea Synthesis by Ruminal Epithelial and Duodenal Mucosal Cells from Growing Sheep*, ${ }^{\dagger}$
}

\author{
M. Oba, ${ }^{1}$ R. L. Baldwin VI, ${ }^{2}$ S. L. Owens, ${ }^{1}$ and B. J. Bequette ${ }^{1}$ \\ ${ }^{1}$ Department of Animal and Avian Sciences, \\ University of Maryland, College Park 20742 \\ ${ }^{2}$ Functional Genomics Laboratory, \\ Animal and Natural Resources Institute USDA-ARS, \\ Beltsville, MD 20705
}

\section{ABSTRACT}

The objective was to determine whether ruminant gut tissues have the capability to synthesize urea in a short-term incubation. Mixed primary cell cultures containing ruminal epithelial (REC) or duodenal mucosal cells (DMC) were isolated from growing sheep $(\mathrm{n}=$ 4) fed a mixed forage-concentrate diet. Cells were incubated (90 $\mathrm{min}$ ) in a Krebs salts-based buffer with either acetate $(5 \mathrm{mM})$ or propionate $(5 \mathrm{~m} M)$ plus a combination of substrate intermediates $(5 \mathrm{mM})$ for urea synthesis: arginine, aspartate + citrulline (AspC), aspartate + ornithine + ammonia (AspON), or AspON $+N$-carbamoylglutamate (AspONG) in a $2 \times 4$ factorial arrangement of treatments. Volatile fatty acid, propionate vs. acetate, did not influence net urea synthesis. For REC, net urea synthesis $\left(\right.$ nmoles $\left.\cdot\left(10^{6} \text { cells }\right)^{-1} .90 \mathrm{~min}^{-1}\right)$ was greatest with Arg (54.5 \pm 6.3) followed by AspC (4.6 \pm 1.1) and AspONG (3.6 \pm 1.4$)$. For DMC, net urea synthesis for Arg (2.1 \pm 0.7$)$ and AspONG (1.9 \pm 0.7$)$ treatments was greater than for AspC ( $0.3 \pm 0.7)$ and AspON (-0.6 \pm 0.7 ) treatments. Thus, for both REC and DMC, arginase activity appeared to be sufficient for catabolism of arginine to urea. Furthermore, greater urea synthesis from ammonia, ornithine and aspartate in the presence of the $\mathrm{N}$-acetylglutamate analogue suggests that carbamoyl phosphate synthetase is probably rate-limiting for urea synthesis and ammonia detoxification by ruminant gut tissues.

(Key words: duodenum, rumen, sheep, urea synthesis)

Abbreviation key: AspC = aspartate + citrulline; AspON = aspartate + ornithine + ammonia $;$ AspONG =

Received September 10, 2003.

Accepted December 16, 2003.

Corresponding author: B. J. Bequette; e-mail: bbequette@ wam.umd.edu.

* Mention of trade name, propriety product, or specific equipment does not constitute a guarantee or warranty by USDA and does not imply its approval to the exclusion of other products that may be suitable.

The authors gratefully acknowledge D. Hucht and M. Niland for technical assistance.
AspON $+N$-carbamoylglutamate, $\mathbf{D M C}=$ duodenal mucosal cells; REC $=$ ruminal epithelial cells.

In ruminants, the poor efficiency of converting dietary protein into milk or muscle protein results partly from the extensive degradation of protein in the rumen with, in consequence, high rates of ammonia absorption and significant excretion of nitrogen in urine (Reynolds et al., 1991). Limiting the breakdown of protein in the rumen, maximizing microbial protein synthesis, and enhancing the recycling of blood urea to the rumen are research areas that have been given the most attention. Observations in the rat (Mouille et al., 1999) and weaned pig (Wu, 1995; Bush et al., 2002) indicate that the colon and intestines possess the necessary complement of enzymes for citrulline and urea synthesis, suggesting that the gut tissues have an ability to detoxify absorbed ammonia. However, it is not known whether the ruminant gut tissues also possess the ability to synthesize urea from absorbed ammonia. If such a mechanism exists in ruminant gut tissues, in particular the rumen epithelia, then this would provide an attractive target for reducing the potential toxic side effects of ammonia absorption, and a target for promoting the local recycling of urea to the rumen for microbial protein synthesis. Thus, the first objective of the current study was to determine whether ruminant gut tissues (rumen and duodenum) synthesize urea. Carbamoyl phosphate synthetase is the first enzyme in the pathway for urea synthesis, and $N$-acetylglutamate is the essential allosteric activator of this enzyme. In addition, propionate has been shown to be a negative regulator of ureagenesis in ruminant hepatocytes (Mutsvangwa et al., 1997). Therefore, an additional objective of our study was to determine whether $N$-carbamoylglutamate (a nonmetabolizable analogue of $N$-acetylglutamate) and propionate act as regulators of urea synthesis by ruminant gut cells.

Mixed primary cell cultures containing ruminal epithelial cells (REC) or duodenal mucosal cells (DMC) were isolated from 4 growing Polypay ram lambs (33.1 
$\pm 4.8 \mathrm{~kg} \mathrm{BW})$. Lambs were fed a pelleted diet, which is comprised of $55 \%$ alfalfa hay and $45 \%$ concentrate (Oba et al., 2004) to meet nutrient requirements ad libitum for at least $2 \mathrm{wk}$ before slaughter. All animal procedures were approved by the Beltsville Agricultural Research Center Institutional Animal Care and Use Committee (protocol \#02-008). Gut cells were isolated separately for each sheep following the procedures described by Baldwin and McLeod (2000) and Oba et al. (2004). Cell viability averaged $73.1 \%$ for REC and $82.4 \%$ for DMC. Immediately after isolation, cells were incubated for $90 \mathrm{~min}$ in media containing either acetate $(5 \mathrm{mM})$ or propionate $(5 \mathrm{mM})$ plus one of four combinations of substrate intermediates in the ornithine-urea cycle (5 $\mathrm{mM}$ each): Arg, aspartate + citrulline (AspC), aspartate + ornithine + ammonium chloride (AspON), or AspON $+\mathrm{N}$-carbamoylglutamate (AspONG). N-Carbamoylglutamate is a stable analog of $N$-acetylglutamate, an allosteric stimulator of carbamoyl phosphate synthetase. Treatments were arranged in a $2 \times 4$ factorial design, and performed in triplicate incubations for each treatment combination.

The incubation media [2.5 mL; Krebs salts (Krebs and Henseleit, 1932) plus $25 \mathrm{~m} M$ HEPES and $0.12 M$ sodium bicarbonate] was oxygenated with $\mathrm{O}_{2}: \mathrm{CO}_{2}(95: 5)$ and the $\mathrm{pH}$ adjusted to 7.4. Incubations were initiated by addition of $0.5 \mathrm{~mL}$ of cell suspension $\left(1 \times 10^{7}\right.$ viable cells) to freshly gassed (20 s under 95:5 $\mathrm{O}_{2}: \mathrm{CO}_{2}$ ) media, and flasks were placed into a reciprocal action shaking water bath at $37^{\circ} \mathrm{C}$. After $90 \mathrm{~min}, 0.2 \mathrm{~mL}$ of concentrated $\mathrm{HClO}_{4}$ was injected into the flasks to terminate the incubation, followed by the addition of $0.3 \mathrm{~mL}$ of $5.8 \mathrm{M} \mathrm{K}_{2} \mathrm{CO}_{3}$ to neutralize the media. Incubation media were then centrifuged $(2300 \times g$ for $7 \mathrm{~min})$, and a known weight $(2 \mathrm{~g})$ of clarified supernatant was added to a known weight $(0.5 \mathrm{~g})$ of $0.15 \mathrm{mM}\left[{ }^{15} \mathrm{~N}_{2}\right]$ urea for determination of urea concentration by isotope dilution. Urea was isolated by cation exchange chromatography (Lobley et al., 1995) and converted to the $t$-butyldimethylsilyl derivative (Calder and Smith, 1988). Then, urea concentration was determined by isotope dilution with gas chromatography-mass spectrometry (HP6890 coupled to an HP5973 Mass Selective Detector, Agilent, Palo Alto, CA) essentially according to the method of Calder et al. (1999). Inter- and intraassay coefficient of variations for urea concentration were 3.8 and $0.5 \%$, respectively. Net urea production was calculated by subtracting background urea present at time 0 from concentrations after 90-min incubation. Data were analyzed separately for REC and DMC using the Proc Mixed procedure of SAS (SAS Inc., Cary, NC) with fixed effect of VFA type, substrate combination, their interactions, and random effect of animals in the model.
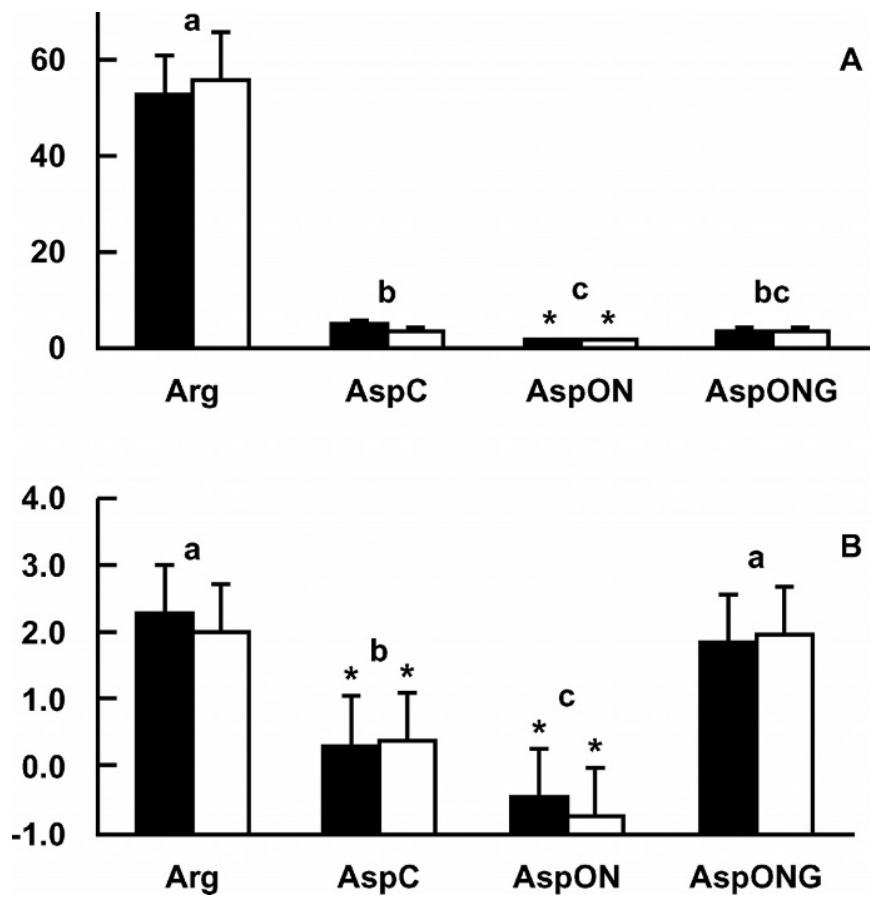

Figure 1. Net urea production (nmoles $\left.\cdot\left(10^{6} \text { cells }\right)^{-1} \cdot 90 \mathrm{~min}^{-1}\right)$ by ruminal epithelial (A) and duodenal mucosal cells (B) incubated with either acetate ( $5 \mathrm{~m} M$, solid bars) or propionate ( $5 \mathrm{~m} M$, open bars) plus one of four combinations of ornithine-urea cycle substrate intermediates [5m $M$ each; arginine (Arg), aspartate + citrulline (AspC), aspartate + ornithine + ammonia (AspON), or AspON + N-carbamoylglutamate (AspONG)]. Paired bars bearing different superscript letters are significantly different from each other $(P<0.05)$. *Net urea synthesis was not different from zero.

Background urea present at time 0 was 2.2 and 2.1 nmoles per $10^{6}$ cells for REC and DMC, respectively. Net urea production was not affected by VFA type, and no interactions between VFA type and substrate combinations were observed. For REC (Figure 1A), net urea production (nmoles $\cdot\left[10^{6} \text { cells }\right]^{-1} \cdot 90 \mathrm{~min}^{-1}$ ) was highest $(P<0.001)$ for $\operatorname{Arg}(54.5 \pm 6.3)$ compared with other treatments. Net urea production was similar for AspC $(4.6 \pm 1.1)$ and AspONG $(3.6 \pm 1.4)$ treatments, and AspONG treatment tended $(P=0.11)$ to be greater than AspON $(1.6 \pm 0.8)$, in which net urea production was not different from zero. For DMC (Figure 1B), net urea production was greater $(P<0.001)$ for $\operatorname{Arg}(2.1 \pm 0.7)$ and AspONG (1.9 \pm 0.7) treatments compared with AspC (0.3 \pm 0.7$)$ and AspON (-0.6 \pm 0.7$)$ treatments, which did not differ from zero.

In the current experiment, we demonstrated that ruminant gut tissues have the capability to produce urea in vitro. Although REC and DMC were incubated with substrate intermediates of the ornithine-urea cycle, our results do not preclude the possibility that urea was also produced from endogenous substrates. However, the stimulatory effect of $\mathrm{N}$-carbamoylglutamate addi- 
tion on urea synthesis by DMC and its tendency by REC suggest that ammonia nitrogen can be utilized in the ornithine-urea cycle if carbamoyl phosphate synthetase is activated. Carbamoyl phosphate synthetase may be a limiting step for ammonia detoxification by ruminant gut tissues just as it is for the liver (Meijer et al., 1985). If urea synthesis from absorbed ammonia can be up-regulated in the ruminal epithelia in vivo, then the local recycling of urea- $\mathrm{N}$ to the rumen for microbial protein synthesis should lead to an increase in overall nitrogen efficiency by ruminants (Lapierre and Lobley, 2001). Thus, urea synthesis in ruminant gut tissues could provide an additional target to reduce ammonia absorption and improve nitrogen utilization.

It is noteworthy that in the presence of Arg, the direct precursor for urea synthesis, net urea production by REC was 25-fold greater than for DMC. This observation is compatible with greater arginase activity in the rumen than small intestinal tissues from sheep and cattle (Aminlari and Vaseghi, 1992). Such high rates of arginine catabolism to urea and ornithine may be linked to the need for ornithine to support polyamine synthesis for maintenance of high rates of cell division and differentiation (Cynober et al., 1995). Alternatively, ornithine may be required for detoxification of ammonia-N via citrulline synthesis (Mouille et al., 1999). Significant arginase activity in ruminal epithelia may also indicate that extensive amounts of nitrogen are recycled to the rumen via arginine. As gut-derived citrulline is a substrate for arginine synthesis by the kidneys (Windmueller and Sapeth, 1981), if ammonia$\mathrm{N}$ is incorporated into citrulline by ruminant gut tissues, the extensive degradation of arginine to urea in REC would contribute to existing inter-organ (gut tissues - kidney - ruminal epithelia) metabolic pathways which recycle nitrogen to the rumen.

Regulation of the ornithine-urea cycle in ruminant gut tissues remains to be elucidated. There is some indication that propionate can reduce urea synthesis in the sheep liver (Mutsvangwa et al., 1997); however, propionate had no effect on urea synthesis by either REC or DMC compared with incubation with acetate in the current experiment. Bush et al. (2002) showed that somatotropin injection had opposite effects on the activity of ornithine cycle enzymes in enterocytes compared with those in the liver, suggesting that regulation of this pathway in gut tissues may differ from that in the liver. Dose-response effects of ammonia on metabolic capacity of ornithine-urea cycle enzymes in gut tissues also require further investigation. Elucidation of the regulatory mechanisms for ammonia detoxification and urea synthesis by gut tissues is important to improve nitrogen utilization in ruminants.

\section{REFERENCES}

Aminlari, M., and T. Vaseghi. 1992. Arginase distribution in tissues of domestic animals. Comp. Biochem. Physiol. 103B:385-389.

Baldwin, R. L., and K. R. Mcleod. 2000. Effects of diet forage:concentrate ratio and metabolizable energy intake on isolated rumen epithelial cell metabolism in vitro. J. Anim. Sci. 78:771-783.

Bush, J. A., G. Wu, A. Suryawan, H. V. Nguyen, and T. A. Davis. 2002. Somatotropin-induced amino acid conservation in pigs involves differential regulation of liver and gut urea cycle enzyme activity. J. Nutr. 132:59-67.

Calder, A. G., and A. Smith. 1988. Stable isotope ratio analysis of leucine and ketoisocaproic acid in blood plasma by gas chromatography/mass spectrometry. Use of tertiary butyldimethylsilyl derivatives. Rapid Commun. Mass Spectrom. 2:14-16.

Calder, A. G., K. E. Garden, S. E. Anderson, and G. E. Lobley. 1999. Quantitation of blood and plasma AA using isotope dilution electron impact gas chromatography/mass spectrometry with U-C13 AA as internal standards. Rapid Commun. Mass Spectrom. 13:2080-2083.

Cynober, L., J. L. Boucher, and M. P. Vasson. 1995. Arginine metabolism in mammals. J. Nutr. Biochem. 6:402-413.

Krebs, H. A., and K. Henseleit. 1932. Untersuchungen über die Harnstoffbildung im Tierkörper in Hoppe-Seyler's Zeitschrift für Physiol. Chemie 210:33-66.

Lapierre, H., and G. E. Lobley. 2001. Nitrogen recycling in the ruminant: A review. J. Dairy Sci. 84 (E. Suppl.):E223-E236.

Lobley, G. E., A. Connell, M. A. Lomax, D. S. Brown, E. Milne, A. G. Calder, and D. A. Farningham. 1995. Hepatic detoxification of ammonia in the ovine liver: Possible consequences for amino acid catabolism Br. J. Nutr. 73:667-685.

Meijer, A. J., C. Lof, I. C. Ramos, and A. J. Verhoeven. 1985. Control of ureogenesis. Eur. J. Biochem. 148:189-196.

Mouille, B., E. Morel, V. Robert, G. Guihot-Joubrel, and F. Blachier. 1999. Metabolic capacity for L-citrulline synthesis from ammonia in rat isolated colonocytes. Biochim. Biophys. Acta 1427:401-407.

Mutsvangwa, T., J. G. Buchanan-Smith, and B. W. McBride. 1997. Effects of ruminally degradable nitrogen intake and in vitro addition of ammonia and propionate on the metabolic fate of L-[1$\left.{ }^{14} \mathrm{C}\right]$ alanine and L- $\left[{ }^{15} \mathrm{~N}\right]$ alanine in isolated sheep hepatocytes. J. Anim. Sci. 75:1149-1159.

Oba, M., R. L. Baldwin, and B. J. Bequette. 2004. Oxidation of glucose, glutamate, and glutamine by isolated ovine enterocytes in vitro is decreased by presence of other metabolic fuels. J. Anim. Sci. 82: in press. $479-486$.

Reynolds, C. K., H. F. Tyrrell, and P. J. Reynolds. 1991. Effects of diet forage-to-concentrate ratio and intake on energy-metabolism in growing beef heifers: Whole body energy and nitrogen balance and visceral heat production. J. Nutr. 121:994-1003.

Windmueller, H. G., and A. E. Spaeth. 1981. Source and fate of circulating cirulline. Am. J. Physiol. 241:E473-E480.

$\mathrm{Wu}, \mathrm{G} .1995$. Urea synthesis in enterocytes of developing pigs. Biochem. J. 312:717-723. 